\title{
Hope to Reality: The Future of Hospitalists and Palliative Care
}

\author{
Steven Z. Pantilat, MD, MHM, FAAHPM*
}

Department of Medicine, University of California, San Francisco, San Francisco, California.

In the inaugural issue of the Journal of Hospital Medicine, Diane Meier made a strong case for palliative care in hospitals ${ }^{1}$ and I advocated for a close partnership between hospital medicine and palliative care. ${ }^{2}$ Over the past decade, hospital medicine and palliative care have grown up together, like fraternal twins, driven by similar demographic trends and imperatives to improve value. In the early going, each sibling played to its strengths: hospital medicine to quality and palliative care to the patient experience. Today, with patient satisfaction a key metric for reimbursement, hospital medicine is focusing on patient experience and may learn from palliative care. Similarly, as palliative care has matured as a field, palliative care teams are being asked to demonstrate quality and may learn much from working with hospital medicine.

Increasingly, palliative care teams are focusing on quality improvement (QI) and collecting the data necessary to drive it. ${ }^{3}$ In fact, there is sufficient interest in QI that 2 Web-based, nationally available databases for collecting outcome data in palliative care have been established, the Palliative Care Quality Network (PCQN; www.pcqn.org) and the Quality Data Collection Tool (qdact; www.qdact.org). ${ }^{4}$ Both systems have created standardized datasets that include patient-level outcomes that palliative care teams collect for every patient and Web-based databases to store the data and produce reports. The data and reports allow palliative care teams to benchmark patient-level outcomes to other palliative care teams, identify best practices, and drive QI. The PCQN, for example, offers intensive education in QI, ongoing QI collaboratives with monthly conference calls, and in-person conferences to help palliative care teams engage in QI. ${ }^{5}$

Although palliative care teams are engaging in QI, there is also the recognition that their efforts effect only those patients referred to their service and that they cannot meet all the need for palliative care in the hospital. Most palliative care teams are struggling to cope with the volume of consults, yet estimate that they are seeing

*Address for correspondence and reprint requests: Steven Z. Pantilat, MD, University of California, San Francisco, 521 Parnassus Ave, Suite C-126, Box 0131, San Francisco, CA 94143-0131; Telephone: 415-4769019; Fax: 415-476-5020; E-mail: stevep@medicine.ucsf.edu

Additional Supporting Information may be found in the online version of this article.

Received: April 20, 2015; Revised: April 30, 2015; Accepted: May 4, 2015

2015 Society of Hospital Medicine DOI 10.1002/jhm.2401

Published online in Wiley Online Library (Wileyonlinelibrary.com). fewer than half the patients who need to be seen. ${ }^{6}$ True quality will require not only that palliative care teams improve the care they provide, but that each hospital and healthcare system adopt a population-based approach to addressing palliative care needs. We will need to implement methods for identifying all patients with palliative care needs, not just the ones referred to palliative care teams, and create systems to meet those needs. Hospitalists and hospital medicine are critical partners in the effort to improve quality for the population of people with serious illness.

An essential aspect of this population-based approach is recognizing that although some patients will have palliative care needs best addressed by a palliative care team of experts, others will have palliative care needs that can be met well by hospitalists, nurses, social workers, and chaplains who have the necessary skills to provide what has been called primary palliative care. ${ }^{7}$ Working together, hospitalists and palliative care teams must develop screening tools integrated within the electronic health record (EHR) to identify patients with palliative care needs such as for completing an advance directive or pain management. ${ }^{8}$ The Society of Hospital Medicine (SHM) has supported similar efforts focused on venous thromboembolism prophylaxis and transitions of care. Identifying palliative care issues proactively will ensure that they are not overlooked. In addition, there must be a system to address those needs. Hospitalists will play a key role in addressing primary palliative care needs. Many hospitalists through study, practice, and focus will develop expertise beyond primary palliative care and be able to address more complex patient needs. Our professional society, the SHM, could collaborate with the American Academy of Hospice and Palliative Medicine to develop a formal recognition for hospitalists who demonstrate skill and focus in palliative care as a way to ensure expertise, expand the cadre of physicians with palliative care skills, and encourage hospitalists to adopt this focus.

Finally, we will need to implement systems to collect key data on palliative care outcomes for all patients. For example, within the EHR we must be able to easily locate a goals of care discussion and its outcome as well as an advance directive. Furthermore, we should work to establish statewide and even national repositories of advance directives so that they are available when needed. Currently the PCQN and qdact collect such data on advance care planning and completion of advance directives, and can assess their impact on key patient outcomes. These organizations 
can collaborate on the solutions for measuring palliative care outcomes across a population. As an example, our palliative care team at UCSF is using PCQN data to drive a QI project focused on increasing the completion of Physician Orders for Life-Sustaining Treatment forms for people seen by the palliative care team who are discharged and whose code status is anything other than full code. Our hospitalist group will adopt this QI project in the coming year based on recognition of its success and importance to the broader population of patients cared for by hospitalists. Such partnerships at the local and national level can improve quality in many dimensions of care including pain management and transitions. Addressing population-based palliative care needs through a partnership between palliative care and hospital medicine will also allow us to answer many questions about the demographics of palliative care needs, the impact of various interventions for addressing needs on patient outcomes, and which patients need expert level palliative care among others.

Ten years ago I wrote that hospitalists and palliative care were a partnership for hope. Growing recognition of the benefits of palliative care, rising patient demand for palliative care, and an increasing focus on
QI are quickly outstripping the ability of palliative care teams to provide that care on their own. To achieve the best patient care, that partnership must be more than hope. It must be a reality.

Disclosures: Dr. Pantilat's work is supported by grants from the Alafi Family Foundation, the Hellman Foundation, the UniHealth Foundation, the California HealthCare Foundation, the Archstone Foundation, and the Kettering Family Foundation. The author reports no conflicts of interest.

\section{References}

1. Meier DE. Palliative care in hospitals. J Hosp Med. 2006;1(1):21-28.

2. Pantilat SZ. Palliative care and hospitalists: a partnership for hope. J Hosp Med. 2006;1(1):5-6.

3. Kamal AH, Hanson LC, Casarett DJ, et al. The quality imperative for palliative care. J Pain Symptom Manage. 2015;49(2):243-253.

4. Casarett DJ, Dy S, Spence C, Lupu D. Foreword: quality improvement efforts: advancing the science of palliative care. J Pain Symptom Manage. 2011:42(5):649-651.

5. Pantilat SZ, Bragg AR, O'Riordan DL, et al. Palliative Care Quality Network: toward quality improvement in palliative care. J Palliat Care. 2014;30(3):204-205.

6. Pantilat SZ, Kerr KM, Billings JA, Bruno KA, O’Riordan DL. Palliative care services in California hospitals: program prevalence and hospital characteristics. J Pain Symptom Manage. 2012;43(1):39-46.

7. Quill TE, Abernethy AP. Generalist plus specialist palliative carecreating a more sustainable model. N Engl J Med. 2013;368(13): $1173-1175$.

8. Glajchen M, Lawson R, Homel P, Desandre P, Todd KH. A rapid two-stage screening protocol for palliative care in the emergency department: a quality improvement initiative. J Pain Symptom Manage. $2011 ; 42(5): 657-662$. 